\title{
Dynamics of the superfine structure in the Orion KL Jet
}

\author{
Leonid I. Matveyenko \\ Space Research Institute, Profsojuznaja 84/32, 117810 Moscow, Russia
}

Phil J. Diamond

Nuffield Radio Observatory, Macclesfield, Cheshire SK11, 9DL, UK

David A.Graham

Max-Planck-Institut für Radioastronomie, Auf dem Hügel 69, 53121, Bonn, Germany

\begin{abstract}
We have studied the superfine structure of the active $\mathrm{H}_{2} \mathrm{O}$ maser region in Orion KL with an angular resolution of $\leq 0.3 \times 0.7$ mas. The high level of $\mathrm{H}_{2} \mathrm{O}$ maser emission from 1979-1988 was due to an accretion disk, which is divided into five groups of protoplanetary rings. The peak brightness temperatures of the structures was $T_{\text {peak }}=$ $10^{13-14} \mathrm{~K}$. The region is located in the OMC-1 molecular cloud, $V_{L S R} \simeq$ $7.74 \mathrm{~km} / \mathrm{s}$. The cloud amplifies by more than two orders of magnitude the emission from the structures, whose radial velocities are within the maser window $\pm 0.3 \mathrm{~km} / \mathrm{s}$. Due to this, the velocity of the $\mathrm{H}_{2} \mathrm{O}$ super maser emission is constant. In the quiescent period of 1995 a $6 \mathrm{AU}$ jet was discovered, $P A=-32^{\circ}, T_{b} \simeq 10^{11} \mathrm{~K}$. In 1998 the jet's brightness temperature increased by more than 3 orders of magnitude. Initially the jet's position angle was $P A=-45^{\circ}$, and then changed to $P A=-38^{\circ}$. During the period of decreasing emission in 1999 the jet had changed its form and became a helix, that suggests the precession of the rotation axes. In the central part of the jet there is a compact bright source "the ejector" - with $T_{e j c}=10^{17} \mathrm{~K}$.
\end{abstract}

\section{Introduction}

Star formation in gas-dust complexes is a collective process accompanied by intense $\mathrm{H}_{2} \mathrm{O}$ maser emission. There are 8 active zones in the Orion KL region (Genzel et al. 1978). The maser emission is linearly polarized and variable. Episodically, an extraordinary phenomenon is observed - outbursts of $\mathrm{H}_{2} \mathrm{O}$ supermaser emission. The nature of this process, its connection with protostellar and protoplanetary formations, and the fine structure of the $\mathrm{H}_{2} \mathrm{O}$ supermaser region is the subject of our studies. We have been observing this object for three epochs: the active period 1979-1987, the quiescent period 1995, and the second active period 1998-1999. 


\subsection{The active period from 1979-1987}

One of the active zones at $R A=05^{h} 35^{m} 14 .^{s} 121, D E C=-05^{\circ} 22^{\prime} 36^{\prime \prime} 27,(2000)$ exhibiting outbursts of $\mathrm{H}_{2} \mathrm{O}$ supermaser emission in 1979-1987 has been documented (Abraham et al. 1981, Matveyenko 1981, Garay et al. 1989). The flux densities of the outbursts were $10^{6} \mathrm{Jy} \leq F \leq 8 \cdot 10^{6} \mathrm{Jy}$ at $V_{L S R} \approx 7.6 \mathrm{~km} / \mathrm{s}$. The supermaser emission was dominated by a chain of 5 groups of compact sources, the size of which was $\sim 0.2$ mas or $\sim 0.1 \mathrm{AU}$, and the brightness temperatures were $T_{b} \leq 10^{17} \mathrm{~K}$, (Matveyenko 1981, Matveyenko et al. 1998). The length of the chain was $10.5 \mathrm{AU}$ and the orientation was nearly in the $\mathrm{E}-\mathrm{W}$ direction. The radial velocities of the groups increased from $V=6.45$ to $V=8.75 \mathrm{~km} / \mathrm{s}$ from west to east. The maser emission was linearly polarized with $P \leq 70 \%$. The structure corresponds to an accretion disk separated into protoplanetary rings, seen edge on. The compact sources are characterized by the tangential direction of the rings. The maser emission is concentrated in the azimuth plane. These structures are located in the dense molecular cloud OMC-1, with a velocity of $\mathrm{V}=7.74 \mathrm{~km} / \mathrm{s}$. The cloud medium amplifies, by more than two orders of magnitude, the emission of the structure inside the maser window $\Delta V= \pm 0.3 \mathrm{~km} / \mathrm{s}$. The amplification dominates the value and "stability" of the outburst's velocity. The velocities and relative positions of the components can be represented by Keplerian motion. However, the stellar wind and light pressure or compression of matter accelerating the $\mathrm{H}_{2} \mathrm{O}$ molecules of the maser rings causes the rings to either expand or shrink. In this case for $V_{L S R}=5 \mathrm{~km} / \mathrm{s}, M \approx(0.2-0.5) M_{\odot}$, the inner radius of the disc is $\mathrm{R}=6 \mathrm{AU}, V_{\text {rot }} \approx 5 \mathrm{~km} / \mathrm{s}$ and decreases as $\sim R^{-0.5}$. The expansion velocity is $V_{\text {exp }}=3.8 \mathrm{~km} / \mathrm{s}$ and decreases as $\sim R^{-0.5}$. The disk diameter is $33 \mathrm{AU}$.

\subsection{The quiescent period of $\mathbf{1 9 9 5}$}

The flux density of the supermaser region was $F \simeq 10^{3} \mathrm{Jy}$ in the quiescent period in 1995. The chain of compact components was invisible. But we detected a bipolar outflow: the jet and two compact components, whose brightness temperatures are $T_{\text {peak }} \approx 2 \cdot 10^{12} \mathrm{~K}$ (Fig.1). The jet's brightness temperature was $T_{b} \approx 10^{11} \mathrm{~K}$, and its emission was linearly polarized. The polarization distribution along the jet is shown in Figure 1a, the position angle was $\chi=-45^{\circ}$ (rotated through $90^{\circ}$ ), and $P \leq 50 \%$. The jet's length was $6 \mathrm{AU}$, its thickness was $\leq 0.5 \mathrm{AU}, P A=-45^{\circ}$, and the velocity was $V_{j e t}=7.63 \mathrm{~km} / \mathrm{s}$. The components velocities and polarizations are: $V_{N W}=7.45 \mathrm{~km} / \mathrm{s}, P \leq 12 \%, \chi=-55^{\circ}$ and for $V_{S E}=7.95 \mathrm{~km} / \mathrm{s}, P \leq 45^{\circ}, \chi=-53^{\circ}$. The differential radial velocities were $-0.25 \leq \Delta V \leq 0.25 \mathrm{~km} / \mathrm{s}$. The SE component had a cometary structure of the form: head - tail, Figure 1b (Matveyenko, Diamond, \& Graham 1998, 2000).

\subsection{The active period between 1998-1999}

The $\mathrm{H}_{2} \mathrm{O}$ supermaser emission with $F \leq 6 \cdot 10^{6} \mathrm{Jy}$ and $V \approx 7.7 \mathrm{~km} / \mathrm{s}$, (Kobayashi et al. 2000, Tolmachev 2000) was associated with the increasing brightness temperature of the jet (Matveyenko, Diamond, \& Graham 2001). Between March- 
August, 1998 , the jet's brightness temperature was $T_{\text {jet }} \approx 2 \cdot 10^{15} \mathrm{~K}$. In the central part of the jet there was a compact source, whose brightness temperature was $T_{b} \approx 5 \cdot 10^{16} \mathrm{~K}$ (Fig. 1). This suggests that the source is an "ejector" and the jet is a bipolar outflow. The visible size of the jet is $\sim 7$ mas and its thickness is $\sim 0.1$ mas. The form of the jet is similar to that seen during the quiescent epoch. But the jet's orientation changed to $P A=-45^{\circ}$ in March 98 and $P A=-38^{\circ}$ later (Fig. 1). The brightness changes in the NW part of the jet from April 5 to August 9, 1998 can be explained by movement of the knots with velocities of $V \geq 30 \mathrm{~km} / \mathrm{s}$, (Kobayashi et al. 2000, Matveyenko et al. 2001). The differential radial velocities of the SE and NW parts of the jet was $-0.6 \mathrm{~km} / \mathrm{s} \leq \Delta V \leq 0.6 \mathrm{~km} / \mathrm{s}$. This means that the outflow is located in the plane of the sky, or the visible velocity is limited by the maser window of OMC-1. The structure of the jet in the period of decreasing activity, from January 23 to April 24, 1999 strongly changed (Fig. 1). The bipolar outflow has a helical shape and the differential velocities between the NW and SE parts is $0.15 \mathrm{~km} / \mathrm{s} \leq \Delta V \leq 0.15 \mathrm{~km} / \mathrm{s}$. The brightness temperature of the ejector is $T_{e j c} \sim 3 \cdot 10^{16} \mathrm{~K}$ and $\mathrm{V}=7.45 \mathrm{~km} / \mathrm{s}$. In the period of 1998-1999, bullets were observed in the directions of $P A=-37^{\circ} \pm 5^{\circ}$ and $P A=138^{\circ} \pm 5^{\circ}$.

\section{Conclusions}

- Our studies of the star formation region in the Orion KL show that the $\mathrm{H}_{2} \mathrm{O}$ supermaser emission comes from a complex and highly organized structure: a chain of 5 groups of compact components and a jet.

- The chain is an accretion disk subdivided into protoplanetary rings and the jet is a bipolar outflow. The jet orientation differs from the disk rotation axis at $\sim 35^{\circ}$.

- Compact sources are distributed in a tangential direction to the rings. The maser emision of the rings is concentrated in the azimuth plane.

- The jet's superfine structure has a helical shape.

- The disk's precession changes the visibility of the rings and the jet's structure.

- The surrounding matter, including the $\mathrm{H}_{2} \mathrm{O}$ molecules, are dropping onto the disk and ejected as a bipolar stream.

- The stream collides with the surrounding matter and serves as a pump for maser emission. IR radiation or a protostellar wind stimulates maser emission in the rings. The directivity of the pumping determines the high degree of linear polarization.

- The structures are located in the dense molecular cloud OMC-1, whose velocity is $V \approx 7.74 \mathrm{~km} / \mathrm{s}$. The brightness of the structures, for velocities inside the maser window of $V=7.74 \pm 0.3 \mathrm{~km} / \mathrm{s}$, are amplified by more than two orders of magnitude.

Acknowledgments. One of us L.I.M. wishes to thank the Max-PlanckInstitut für Radioastronomie for support and K.Zaharin for help in preparing the article. 


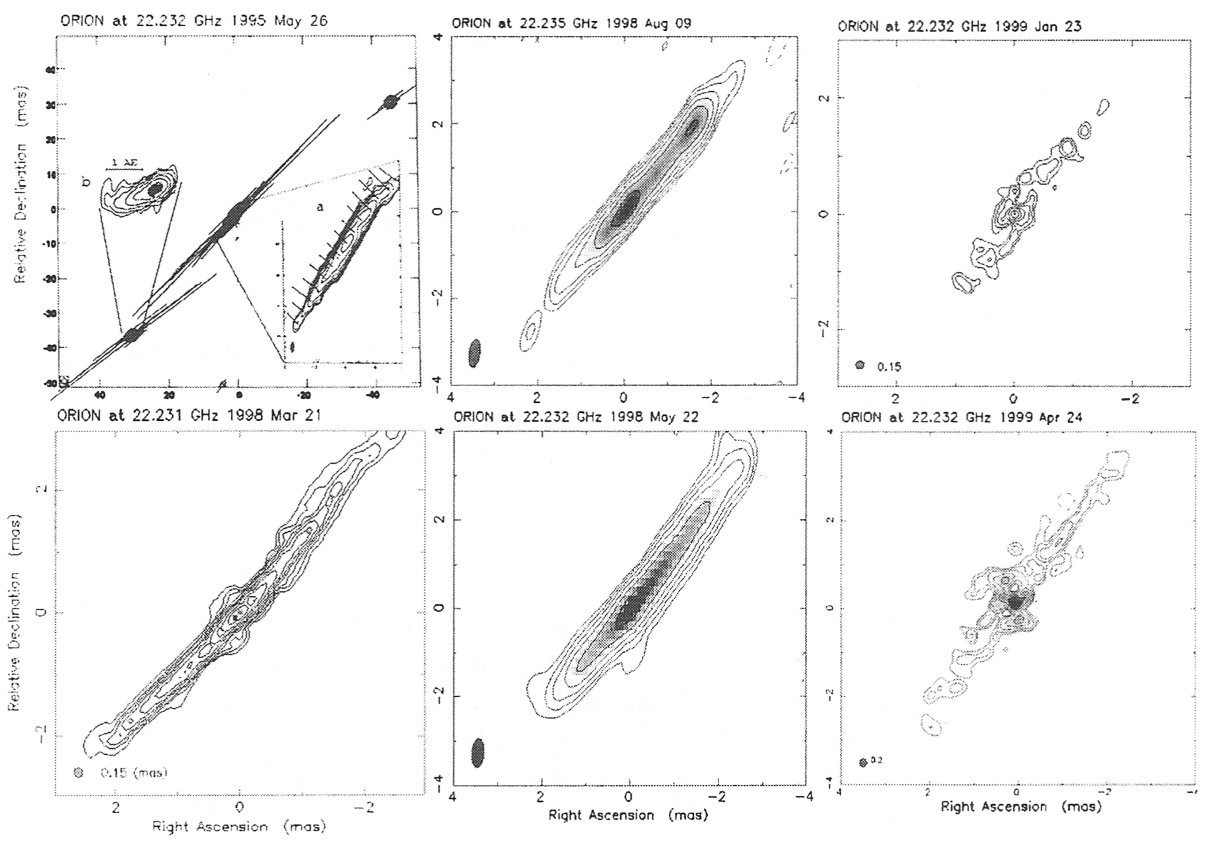

Figure 1. Multi-epoch maps of the $7.6 \mathrm{~km} / \mathrm{s} \mathrm{H}_{2} \mathrm{O}$ super maser region: On 1995 May 26, the quiescent epoch, the beam is $\varphi=0.3 \cdot 0.7$ mas, the structure has a jet and two components, $T_{\text {peak }} \approx 10^{11} \mathrm{~K}$, "a" distribution of polarization in the jet, position angle rotated at $90^{\circ} ;$ " $\mathrm{b}$ " - SE component. On 1998 March $21, \varphi=0.15 \cdot 0.15$ mas, $T_{e j c} \simeq 3 \cdot 10^{16}$ K. On 1998 May $22, \varphi=0.3 \cdot 0.7$ mas, $T_{e j c} \simeq 2 \cdot 10^{16} \mathrm{~K}$. On 1998 Aug 08, $\varphi=0.3 \cdot 0.7$ mas, $T_{e j c} \simeq 5 \cdot 0^{15} \mathrm{~K}$. On $1999 \mathrm{Jan} 23, \varphi=0.15 \cdot 0.15$ mas, $T_{e j c} \simeq 3 \cdot 10^{16} \mathrm{~K}$. On 1999 Apr $24, \varphi=0.2 \cdot 0.2 \mathrm{mas}, T_{e j c} \simeq 3 \cdot 10^{16} \mathrm{~K}$.

\section{References}

Abraham, Z. et al. 1981, A\&A, 100, L10

Garay, G., Moran, J.M., Hashick, A.D. 1989, ApJ, 338, 224

Genzel, R. et al. 1978, A\&A, 66, 13

Kobayashi, H. et al., 2000, in Proc. of the VSOP Symp., 109

Matveyenko, L.I. 1981 SovAL, 7, 100

Matveyenko, L.I., Graham, D.A., Diamond, P.J. 1988, SovAL, 14, 1101

Matveyenko, L.I., Diamond, P.J., Graham, D.A. 1998, SovAL, 24, 723

Matveyenko, L.I., Diamond, P.J., Graham, D.A. 2000, AZh, 9, 869

Matveyenko, L.I., Diamond, P.J., Graham, D.A. 2001, in Proc. IAU Conf. 205

Tolmachev, A.M. 2000, SvAL, 26, 41. 